Results 15 people were excluded from the analysis for both years. The cohort increased from $n=166$ in 2013 to $n=170$ in 2014, with a similar increase in the number of people with Ineb data for $\geq 3$ months ( $\mathrm{n}=83$ in 2013, $\mathrm{n}=85$ in 2014). Median nebuliser adherence improved from $40.5 \%$ in 2013 to $49.5 \%$ in 2014 . The median FEV1 remained stable at $79 \%$ while BMI improved slightly from 22.0 in 2013 to 22.7 in 2014 . The total IV days reduced by 657 from 3970 in 2013 to 3313 in 2014; a potential saving of around $£ 156,000$.

Conclusion Although adherence remains a challenging issue, these data suggest the potential of benefits of improved adherence. More work will be needed to examine the adherence data in more detail and to collect further longitudinal data to determine if there is a clear trend of improvement.

\section{P275 PREVALENCE AND STRAIN TYPING RESULTS OF GRAM- NEGATIVE EMERGING BACTERIAL PATHOGENS IN PATIENTS ATTENDING A LARGE UK ADULT CF CENTRE}

${ }^{1} \mathrm{HD}$ Green, ${ }^{1} \mathrm{R}$ Bright-Thomas, ${ }^{2} \mathrm{D}$ Kenna, ${ }^{1} \mathrm{AM}$ Jones. ' University Hospital of South Manchester, Manchester, UK; ${ }^{2}$ Public Health England, London, UK

10.1136/thoraxjnl-2015-207770.411

Introduction In recent years Gram-negative bacterial emerging pathogens (EP) have been noted to infect the airways of patients with CF. Prevalence of EP is increasing but much remains unknown. This study aimed to determine prevalence of EP at a large adult UK centre and whether these organisms may be capable of cross infection.

Methods Prevalence of Burkhoderia multivorans; Stenotrophomonas maltophilia and Achromobacter; Ralstonia and Pandoraea species was calculated in October 2013 and 2014. Strain typing was performed on EP isolated from patients from January 2008 to present using pulsed-field gel electrophoresis following restriction with XbaI. Epidemiology of patients with shared strains was analysed by reviewing patient addresses, outpatient appointments, admissions, paediatric centres and asking patients about their social behaviours.

Results In October 2013, 358 patients had at least 1 sputum culture result in the previous 12 months and were included in the prevalence calculation. This increased to 368 patients in October 2014. Prevalence of most EP increased between 2013 and 2014. EP prevalence in 2014 ranged from 1.9\% (Ralstonia species) to $6.2 \%$ (B. multivorans) (Table 1). 96 patients had $\geq 1$ isolation of an EP between January 2008 and July 2015. Of these, 20 (21\%) have had $>2$ strains of EP isolated from their sputum within that timeframe. To date, strain typing has been performed on 97 of 115 identified isolates from 96 patients. Shared strains of EP in unrelated patients with epidemiological connexions other than place of home residence were found in cases of infection with Achromobacter, Ralstonia and Pandoraea species. Shared strains of B. multivorans were found in a sibling pair, an unrelated pair with no temporal overlap in positive cultures and in 4 patients with no clear opportunities for cross infection to have occurred. Conclusions Prevalence of EP is low at our centre but is slowly increasing. History of EP infection appears to be a risk factor for infection with other EPs. Shared strains of Achromobacter, Ralstonia and Pandoraea species have been identified in our centre in patients with epidemiological connexions. Numbers are too small to establish whether cross infection or a common environmental source is responsible.
Abstract P275 Table 1 Prevalence of Gram-negative bacterial emerging pathogens at Manchester Adult Cystic Fibrosis Centre 2013 and 2014

\begin{tabular}{|ccc|}
\hline Organism & $\begin{array}{c}\text { Prevalence } \\
\text { October 2013, \% } \\
\text { (number of patients) }\end{array}$ & $\begin{array}{c}\text { Prevalence } \\
\text { October 2014, \% } \\
\text { (number of patients) }\end{array}$ \\
\hline $\begin{array}{c}\text { Burkholderia } \\
\text { multivorans }\end{array}$ & $6.4(23)$ & $6.2(23)$ \\
\hline $\begin{array}{c}\text { Achromobacter } \\
\text { species }\end{array}$ & $4.5(16)$ & $5.1(19)$ \\
\hline $\begin{array}{c}\text { Stenotrophomonas } \\
\text { maltophilia }\end{array}$ & $3.4(12)$ & $4.6(17)$ \\
\hline $\begin{array}{c}\text { Pandoraea } \\
\text { species }\end{array}$ & $2.2(8)$ & $2.4(9)$ \\
\hline $\begin{array}{c}\text { Ralstonia } \\
\text { species }\end{array}$ & $1.4(5)$ & $1.9(7)$ \\
\hline & & \\
\hline
\end{tabular}

P276

THE PREVALENCE OF TICARCILLIN HYPER-SUSCEPTIBLE PSEUDMONOAS AERUGINOSA ISOLATES FROM NON CYSTIC FIBROSIS BRONCHIECTASIS PATIENTS COMPARED TO PATIENTS WITH CYSTIC FIBROSIS AND CONTROLS

${ }^{1}$ IT Hettiarachchi, ${ }^{2} \mathrm{~T}$ O'Sullivan, ${ }^{1} \mathrm{M}$ Wootton, ${ }^{3} \mathrm{~J}$ Duckers, ${ }^{1} \mathrm{R}$ Dhillon. ${ }^{1}$ Public Health Wales, Cardiff, UK; ${ }^{2}$ Cardiff University, Cardiff, UK; ${ }^{3}$ Cystic Fibrosis Centre, University Hospital of Llandough, Cardiff, UK

\subsection{6/thoraxjnl-2015-207770.412}

Background and aims Pseudomonas aeruginosa (PsA) is associated with considerable morbidity and mortality in Non-Cystic Fibrosis bronchiectasis (NCFB) and Cystic Fibrosis (CF) patients. Ticarcillin, a carboxypenicillin, is occasionally used in NCFB and $\mathrm{CF}$ to treat pulmonary exacerbations. In $\mathrm{CF}$, a subpopulation of PsA exists that is hypersusceptible to ticarcillin (Tichs) in vitro, (minimum inhibitory concerntration [MIC] $<4 \mu \mathrm{g} / \mathrm{ml}$ ). This phenotype, is associated with reduced MICs to $\beta$-lactams, fluoroquinolones, tetracyclines and a degree of resistance to aminoglycosides.

The aim of this study was to investigate whether this Tichs strain exists in NCFB patients and compare this to the prevalence rates from $\mathrm{CF}$ and control cohorts. We also assessed whether this strain correlated with enhanced susceptibility to temocillin and other anti-pseudomonal antibiotics.

Methods 18 isolates of PsA from NCFB patients, 23 PsA isolates from $\mathrm{CF}$ patients and 18 PsA isolates from controls with no chronic lung disease were analysed. MICs for each isolate were determined by agar dilution using ISO20776-1 for the antibiotics listed in Table 1 and interpreted using EUCAST breakpoints. Results The NCFB isolates had the highest prevalence of the Tichs strain of the three cohorts we tested, with a prevalence of $76 \%$, compared to a prevalence of $48 \%$ in the CF cohort and $0 \%$ in the controls. Resistant strains of PsA were more prevalent in the CF cohort compared to the NCFB and control cohorts, except for temocillin and ticarcillin where the CF and NCFB cohorts had lower MICs compared to the control cohort (Table 1).

The Tichs strain in NCFB and CF was associated with reduced MICs to all antibiotics apart from ciprofloxacin in comparison to the non- Tichs strain. In CF, the Tichs strain was also associated with increased MICs to gentamicin.

Conclusion Our data supports the existence of a Tichs strain of PsA in NCFB patients, which existed in greater prevalence compared to our CF cohort. This appears to correlate with reduced MICs to temocillin, to which PsA would normally be resistant. 